\title{
Diagnostic benchmarks on dynamic characteristics \\ of thin-walled marine damaged structures
}

doi:10.2478/mape-2019-0004

Date of submission to the Editor: 04/2018

Date of acceptance by the Editor: 07/2018

MAPE 2019, volume 2, issue 1, pp. 38-52

\section{Adam Szeleziński}

ORCID ID: 0000-0003-2842-0683

Lech Murawski

ORCID ID: 0000-0003-0089-5492

\section{Adam Muc}

ORCID ID: 0000-0002-9495-087X

Gdynia Maritime University, Poland

\section{INTRODUCTION}

Among various means of transport, maritime transport (beside the air transport) is exposed to relatively highest risk. Seagoing vessels often work under extremely difficult environmental conditions (Montewka, et. al., 2014, Vizentin and Vukelić, 2017, Senjanović, et. al., 2014). Moreover, maritime constructions (ships, offshore structures) are exposed to the influence of aggressive marine environment for a very long time. The strength and reliability analysis of maritime constructions have to consider the impact made by waves and wind (storms) including the underwater earthquakes. The SHM system should predict the results of collisions, corrosions, and erosion.

Each marine ship works under the supervision of one of the classification societies. They require a detailed periodic safety inspection of the structure from its design to construction. The welded joints are one of the key elements which undergo precise diagnostic testing. All responsible welds are tested using measurement techniques generally called NDT (Non-Destructive Testing) (Masayasu, 2016, Mvola and Suoranta, 2014). Currently, the hybrid tests are becoming the standardized part of NDT. They use mixed approach that combines two or more methods. Hybrid methods are divided into two groups: using the same physical phenomena and acting on the basis of complementary methods of research. An example of a hybrid approach using the same physical phenomenon is the combination of acoustic emission and ultrasound testing. An example of a hybrid approach that is based on acting on the basis of complementary methods of research is surface testing combined with radiographic and/or ultrasonic testing (Runnemalm, 2012, Ongpeng, et. al., 2018). NDT techniques are well tested and give the results which are sufficiently reliable. However, they have one basic damage - they are run periodically. In the intervals between tests, the reliability of the structure is uncertain (Szeleziński, et. al., 2016). Particularly in the case of critical events (extremely strong storm, collision, etc.), it is

\footnotetext{
*a.szelezinski@wm.umg.edu.pl
} 
important to note the possibility of further operation of the facility. This information must include the degree of danger of disasters along with the operational parameters, including the time of its safe use. To this end, the new techniques are being developed. They are known as SHM (Structural Health Monitoring).

Structural Health Monitoring is a natural extension of NDT diagnostics of machines and devices. Monitoring is an interdisciplinary research field which aim is development and practical use of methods to detect and monitor the structural damage by the measurement system which is integrated with the tested device, operating on-line and automatically. Monitoring may be based on the range, often very different, measurement techniques. The most promising techniques in maritime conditions are: methods based on testing of characteristics of dynamic structures, acoustic emission, study of elastic waves of Lamb Waves type along with spectra finite element method, thermovision methods, ultra-fast framing cameras, layered testing of electromagnetic characteristics, vacuum comparative research and the methods based on fiber optic sensors (Abrantes, 2014, Karuse, et. al., 2015, Murawski, et. al., 2012). The aim of SHM systems is to create the measuring and diagnosis system which would be able to assess the technical condition of the structure continuously and in various environmental and operating conditions. The typical SHM system consists of the net of sensors which continuously measure condition of the structure and environmental and operating loads. The task of system's central unit is to collect and record measurement signals, analyze them (including selection) and to automatically diagnose occurring risks (Murawski, et. al., 2012, Opoka, et. al., 2013).

Work on the elements of the structure monitoring meaning: detection, localization and identification of damages, are being intensively developed (Recho, 2012, Jiawei, et. al., 2018, Vukelić and Brnić, 2016, Dae-seung, et. al., 2016) but mostly they are reduced to laboratory and/or preliminary testing. Moreover, shipbuilding works are relatively underdeveloped (e.g. in comparison to aviation). Complete monitoring complements the detection, tracing and fault identification systems by reliable lifetime prediction of the structure and assessments of its further emergency operation. There are no simplistic but reliable mathematical models for static and dynamic evaluation, parameters (reliably relevant for shipbuilding) in marine industry. These models should be able to be used in systems based on artificial intelligence. Evaluation of the key measurement elements and their effective selection for such a system is essential (Szeleziński, et. al., 2017). Monitoring based on vibrodiagnostic techniques is one of the most promising types due to its simplicity and relatively low costs. So far other techniques do not have practical application. For example, tests which use elastic waves require extremely expensive measuring equipment (e.g. 3D laser) difficult to use in operating conditions for such complex construction as the hull of a ship (Szeleziński, et. al., 2017, Muc, et. al., 2018).

The authors of the paper are searching for new parameters - benchmarks, which may be used in practical SHM systems mounted onboard. In the first stage of analysis of research results, the authors, basing on the recorded spectrum of responses have calculated the classical FFT (Fast Fourier Transform) method. The next stage of the research was to assess the rate of dispersion of the spot and the power of impact. Admissible dispersion was then suggested, to which spectral analysis of dynamical characteristics was applied with statistical methods. 
Eponymous diagnostic parameter of thin-walled structure is based on the assumption that damaged construction is nonlinear. The first analysis method take into account distribution of amplitude specters mean value calculated with the method of time windows (statistical measure in the shape of mean value as assumed parameter, of which analysis of a given part of structure may allow for its quality assessment). Time windows method allows for simulation of signals analysis in the field of frequency and time. The second analysis method of the research results is based on calculating the logarithmic decrement, which changes with time together with the change of responses. In the recent paper the authors supplemented the method of quality analysis of the quality of making the welded joints by describing two algorithms of calculating logarithmic decrement. In the authors' opinion, the SHM system can be applied to the practice only when the automatic analyses would be based on several different analyses which can be compared.

\section{MEASUREMENT METHOD}

The test stand to conduct the testing of welded joints using the vibrodiagnostic method was constructed at the Faculty of Marine Engineering at Gdynia Maritime University. The structure of the stand and its most important parts are presented in Fig. 1. The stand includes the holder which can be used to install the plates (welded test pieces) horizontally - 4-point mounting or vertically - 2-point mounting. The measuring system consists of vibration analyzer produced by Bruel \& Kjaer, two accelerometers, modal impact hammer with three interchangeable heads i.e. metal, silicon, and teflon.

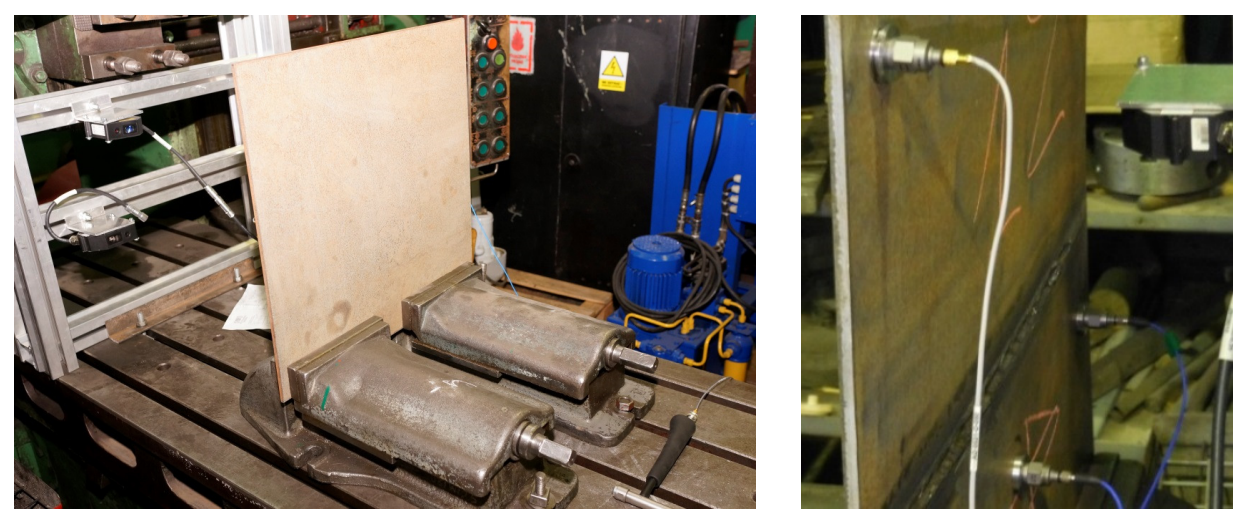

Fig. 1 Measuring stand

During the process of the presented tests, the plates were installed vertically on the holder as it is presented in Fig. 1. The tests were conducted on four plates. The plate marked by number 0 was homogenous and did not have welded joints. The other three included welded plates that were marked by number: 2202 - the plate that did not have any incompatibilities, 2127 - the plate that had incompatibilities in the form of boundary bonding and 2132 - the plate with simulated cracks along the whole length.

All test pieces that have welded joints were tested using the radiographic method before the measurement (see Fig. 2). It enabled the assessment of the joints quality along with identification and placement of incompatibilities in the plates. 


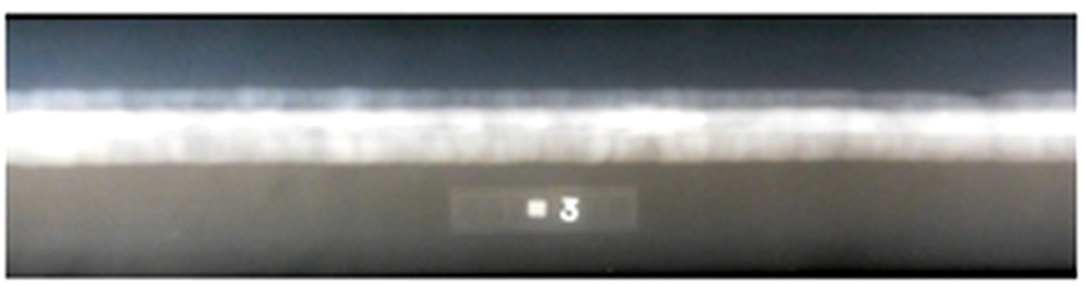

(a)

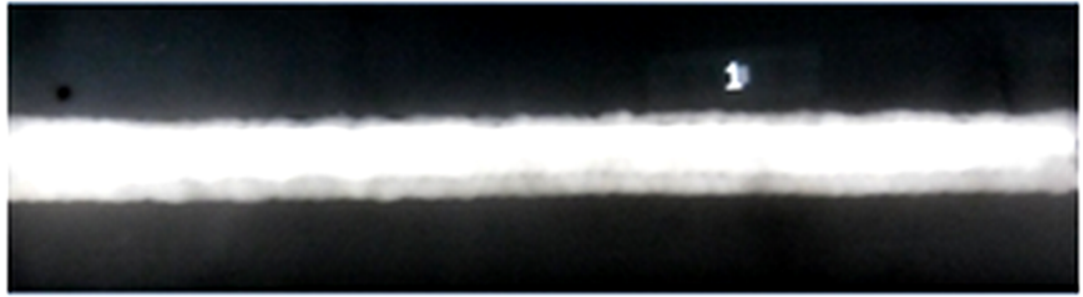

(b)

Fig. 2 Radiographic photography of welded joints where a) welded joint without faults (2202) and

b) welded joint with incompatibility in the form of boundary bonding (2127)

The measurements of vibration generated by the plates were taken in the prepared test stand. The vibration was caused by the impact hammer with different heads: metal, silicon, and teflon. The places of strokes are presented in Fig. 3, described by means of $\mathrm{F} 1, \mathrm{~F} 2$, and F3. The results were read by the accelerometers ACC1 and ACC2.

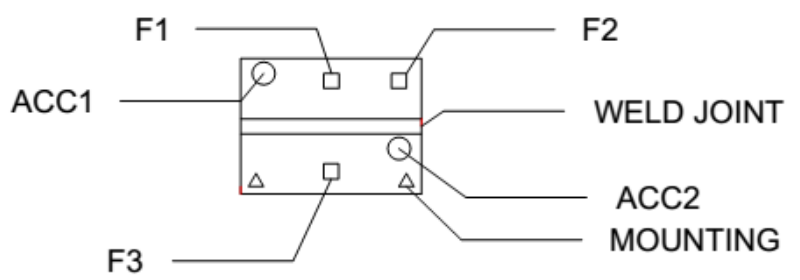

Fig. 3 Schematic diagram showing the arrangement of accelerometers (ACC1, ACC2), places of strokes (F1, F2, F3) and plates mounting places in the holders $(\Delta)$

The concept of a comparative method for the evaluation of welded plates is based on preliminary results obtained by the comparison of amplitude spectras calculated for vibration responses to excitation with different modal hammer ends. Fig. 4 to 6 shows the amplitude spectras for vibrations of tested plates: one with non-defective welds, one with defective edge fusion and one with a cracking along the entire length.

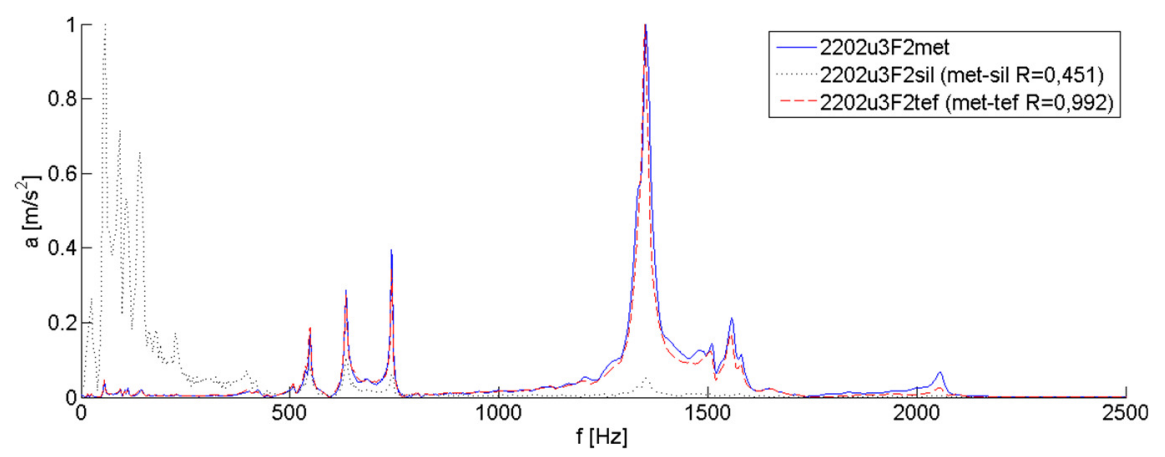

Fig. 4 Amplitude vibration spectra for a plate with a non-defective weld, type 2202 (ends: metal, silicone and teflon) 
The spectral characteristics used were normalized in relation to the highest value obtained for each of the analyzed cases.

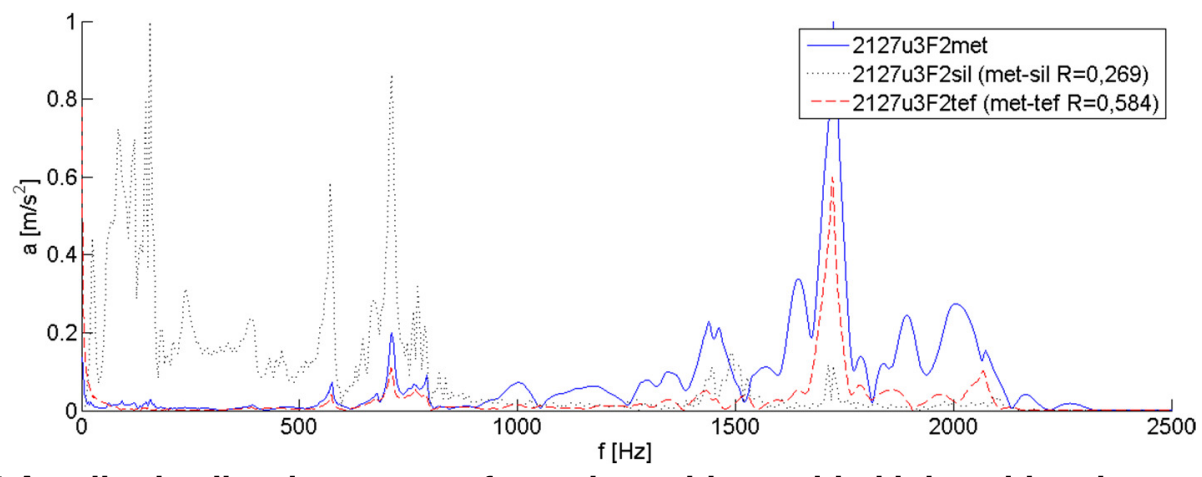

Fig. 5 Amplitude vibration spectra for a plate with a welded joint with a damage type 2127 (metal, silicone and teflon ends)

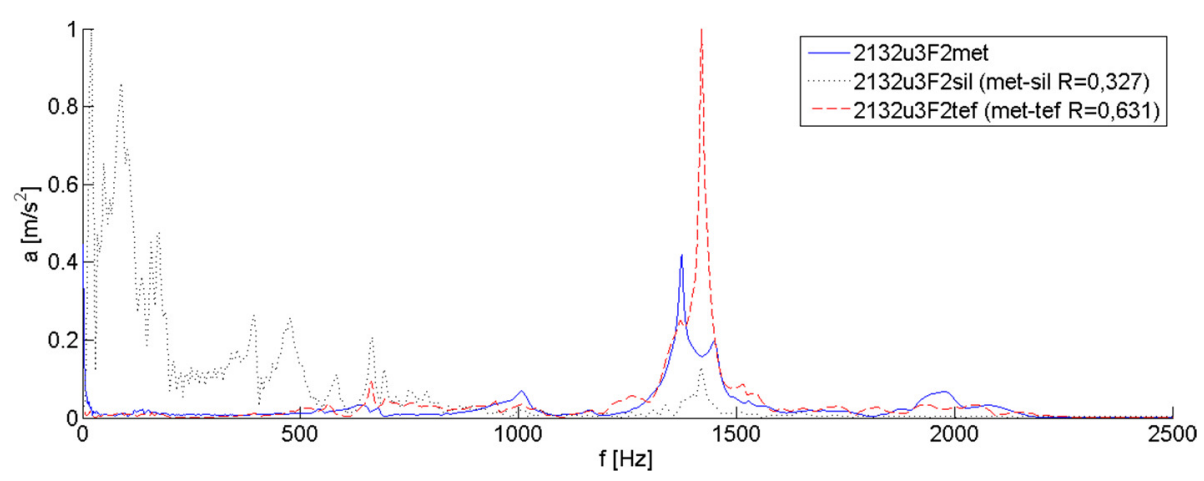

Fig. 6 Amplitude vibration spectra for a plate with a welded joint with a damage type 2132 (metal, silicone and teflon ends)

The spectral characteristics shown in Fig. 4 to 6 were calculated for a non-defective welded plate and for a plate with faulty egde fusion and one with a cracking along its length, and for various modal hammer ends. A comparison of the characteristics shown in Fig. 4 to 6 points to one regularity, i.e. that the results obtained for the silicone end are distinctly different from the results obtained from teflon and metal ends. The amplitude spectra for the silicone end differ greatly in shape from the rest. They are characterized by excessive excitation, especially at lower frequencies. It can also be seen from the presented characteristics that, for a non-defective welded plate, the spectra obtained with teflon and metal ends are very similar, whereas for the defective plates they differ considerably. Apart from the assessment based on a visual comparison of characteristics, calculations were performed to determine the Rsquared coefficient, also called the coefficient of determination. This coefficient can be used to compare and assess the degree to which two characteristics match. In the conducted tests, the spectra obtained for the metal end of the modal hammer were considered as endogenous data, while the other characteristics were seen as exogenous data. The R-squared coefficient assumes values in the interval of $(1 ; 0)$, which means that a coefficient value close to 1 indicates very good compatibility of endogenous and exogenous data. If the case is the opposite, i.e. the coefficient approaches 0 , a poor level of compatibility between the data is indicated. In the analysed cases, it was found that the best match was between results obtained for the metal and teflon ends on the non-defective welded plate, in the other cases the 
value of the determination coefficient was much lower, although it did not exceed 0.5. As a confirmation of the above statement,the authors observed low determination coefficient values for silicone and metal ends (not exceeding 0.5 ).

Comparing presented results, it can be concluded that for the plate with non-defective welding the statistical measures confirm the correlation between the amplitude spectra obtained for the metal and teflon ends. On the other hand, such compatibility was not observed in cases where there was a welding defect, where metal and teflon ends were used. In the next parts of the article, the calculations were made for the measurement results obtained using a metal head installed on a modal hammer.

\section{DAMPING DECREMENT IN WELDED JOINTS ASSESSMENT}

Using the vibrodiagnostic method in tests on welded joints allows analyzing the amplitudes of structure vibrations, in this case, made by modal hammer. While testing, it is possible to record few responses - vibration amplitudes. In order to achieve that, the accelerometers were placed in different location on the tested plate. Recorded responses take the form of damped oscillatory motion. The weld (including its quality), which connects two plates, influences the disappearance speed of diagnostic signal (the speed of energy dissipation), and this is why it is possible to use a damping decrement in its assessment. The dumping decrement is commonly used in assessing the dynamics of many mechanical systems. However, due to the variable nature of response in time, it is impossible to apply regular formula for the logarithmic decrement, expressed as time-independent constant. Two forms of logarithmic decrement were derived in this work and they are represented by formulas 1 and 2 . Statement (1) is used to determine averaged structure damping in regard of maximal amplitude; statement (2) is used to assess the damping changes during single vibration periods or its groups.

$$
\begin{gathered}
\Psi_{I}=\frac{1}{n} \cdot \ln \left(\frac{A_{0}}{A_{n}}\right) \\
\Psi_{I I}=\frac{1}{n-m} \cdot \ln \left(\frac{A_{m}}{A_{n}}\right)
\end{gathered}
$$

where:

$\psi$ - logarithmic decrement,

$A_{0-n, m}$ - subsequent amplitude peak values,

$n, m$-subsequent number of amplitude.

In case of welded joints, damped oscillatory motions of responses recorded by accelerometers are characterized by uneven distribution of maxima and minima. Due to variable changes of vibration speed and the need to calculate damping decrement, the possibility of approximations of response runs was checked using different functions which are generally represented by polynomial and exponential formulas. In order to choose a proper approximating function, the comparative analysis was conducted, in which the approximations obtained by polynomials from second to fifth degree were collated with an exponential function. Fig. 7 and 8 show the exemplary result of approximations for the response recorded for the sample without faults and the sample with the incompatibility in the form boundary bonding while running the tests using a metal head on the modal hammer. 


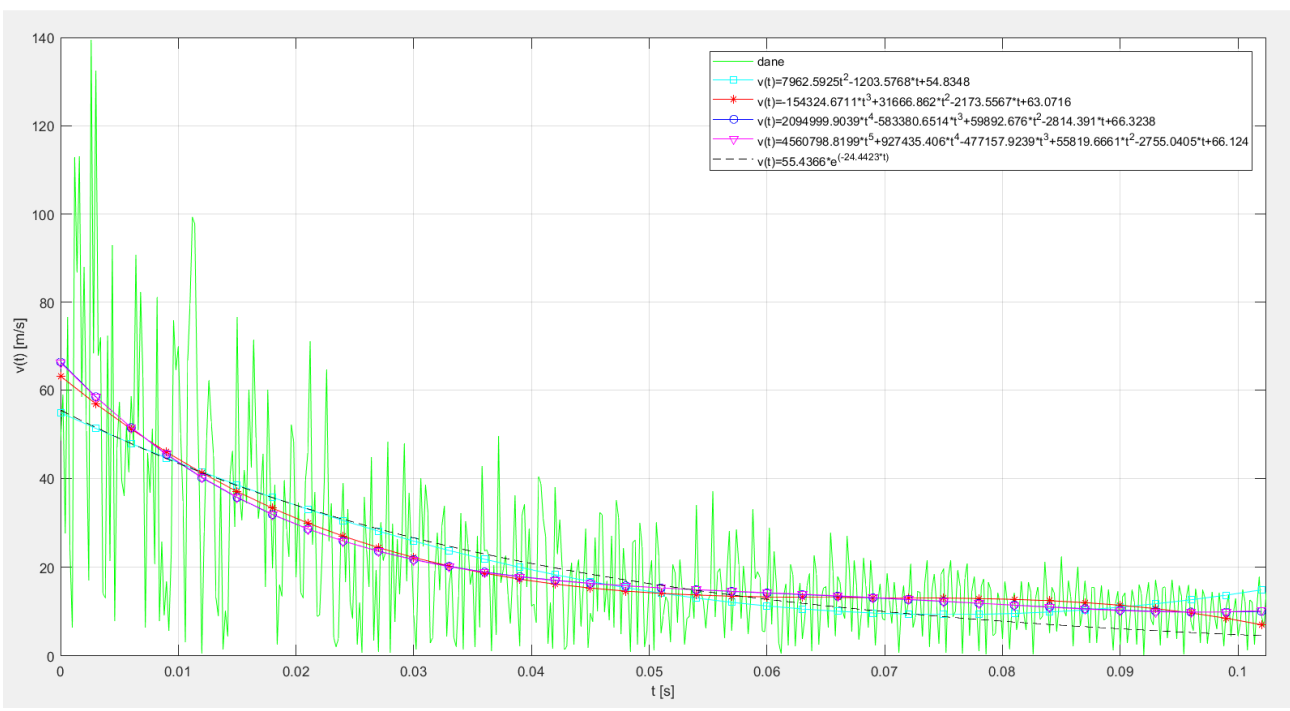

Fig. 7 Example of approximation of the response received for the welded plate without faults (2202)

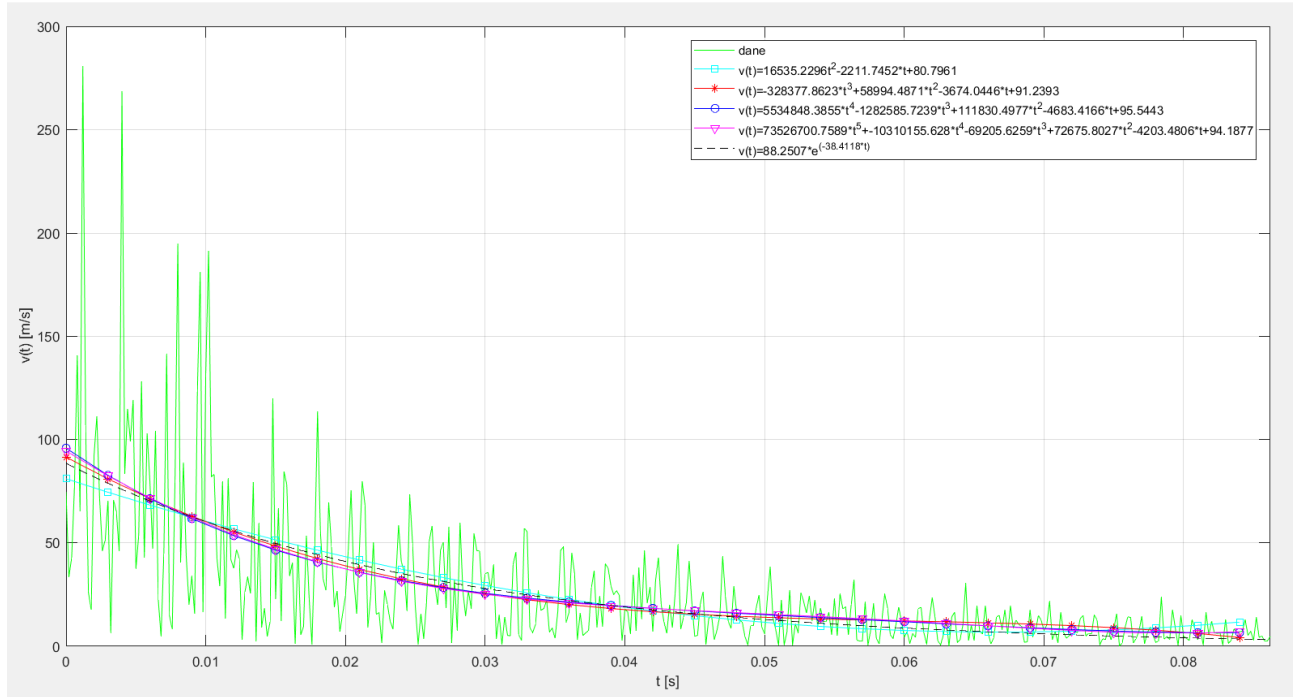

Fig. 8 Example of approximation of the response received for the welded plate with incompatibility in the form of boundary bonding (2127)

By verifying the results of the approximation, it may be concluded that the seconddegree polynomial is not a proper one in the test case because in regard to small amplitudes it deviates from true values of responses increasing the approximation error. However, the other functions allow determining the approximate response in a satisfactory way. At the same time, it can be noticed that increasing the degree of polynomials above 4 does not improve the approximation of received response.

Equations 1 and 2 were used to calculate the damping decrement. The first formula allows calculating the decrement distribution in regard of maximal amplitude, and the second one in regard to chosen range. The following figures present the results obtained for first formula. For the results obtained from each approximating function, the damping decrement was calculated in regard to maximal value of amplitude. The damping decrement obtained with approximation by exponential function is characterized by linear change. Moreover, it was considered to be the reference for other results. 
Fig. 9 present changes of damping decrement for the homogenous plate without welded joint along the different approximating functions. It is noticeable that the damping decrements distributions determined for the plate without the welded joint represent the constant type. It reflects the uniform damping, which is observable for plates showing characteristics of linear structure both materially and geometrically.

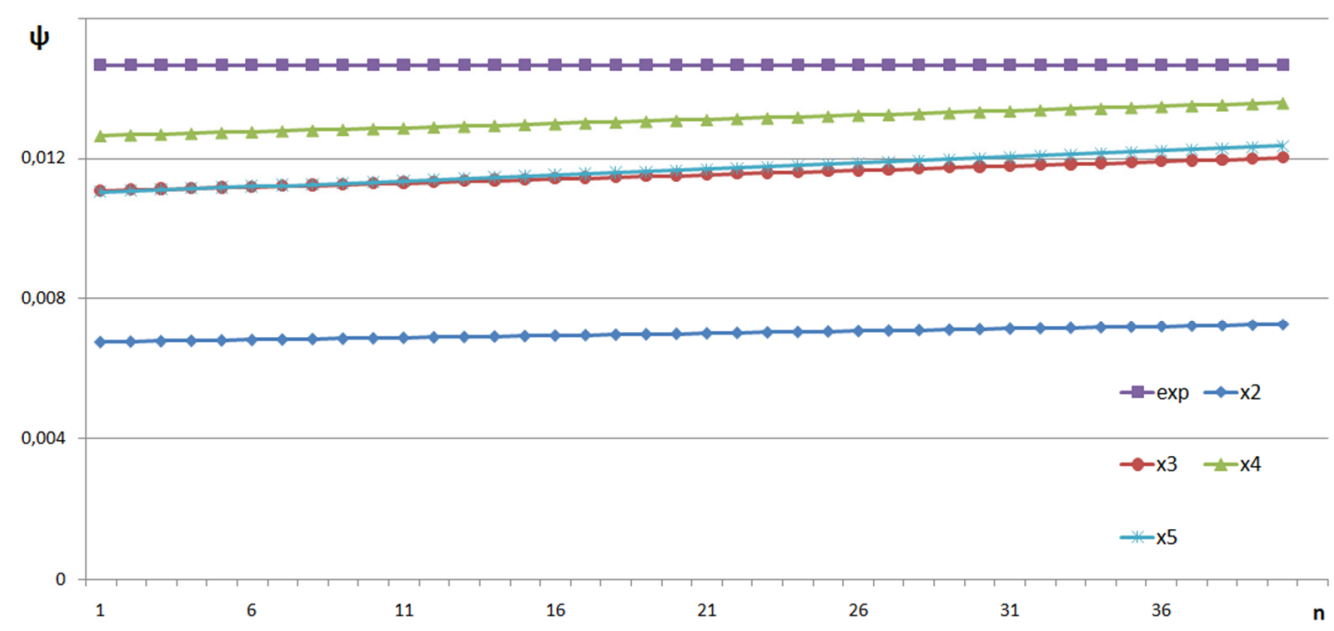

Fig. 9 Values of damping decrement changes for the plate without weld (0)

It is possible to observe higher, time-varying damping for material with a good weld (Fig. 10). Therefore, decrements runs are clearly curved and become concurrent with a decrement calculated from exp function. The intersection with the reference characteristic of the decrement occurs at the $41^{\text {st }}$ peak amplitude of response. This effect is even more noticeable in case of the welded plate with the incompatibility in the form boundary bonding (Fig. 11) because the intersection occurred already at the $31^{\text {st }}$ peak amplitude of the response.

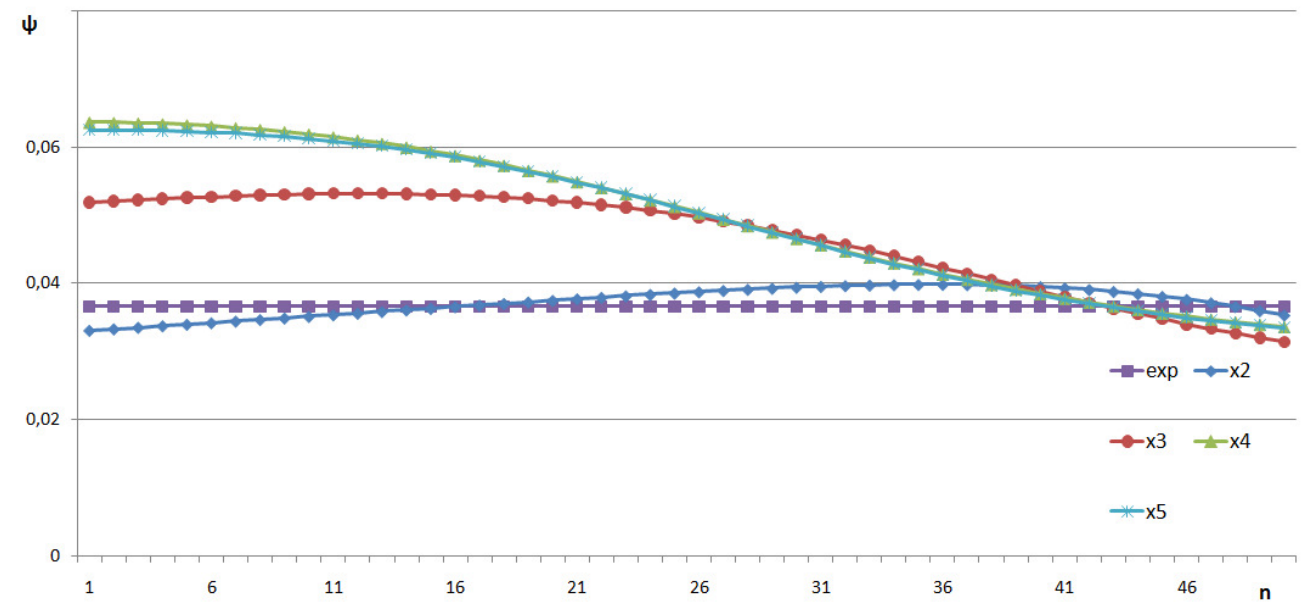

Fig. 10 Values of damping decrement changes for the plate with a weld without faults on a modal hammer (2202) 


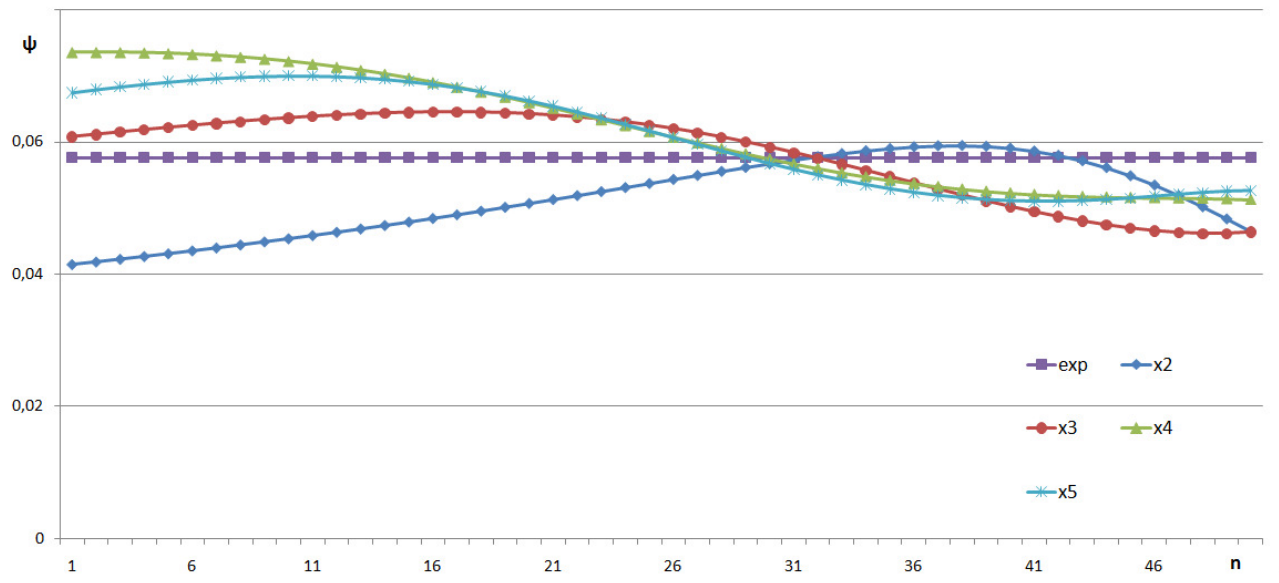

Fig. 11 Values of damping decrement changes in time for the plate with a weld having the incompatibility in the form boundary bonding (2127)

\section{THE ANALYSIS OF TIME-FREQUENCY DYNAMIC CHARACTERISTICS}

The complementation of the amplitude-frequency analysis (FFT) presented in the chapter 2, is its combination with the analysis of the change in vibration amplitudes in the time domain. The calculation procedure has been implemented to present the simultaneous changes of response modules (3) which were recorded by accelerometer in the time and frequency domain.

$$
|X|=g(t, f)
$$

where:

$|X|$ - vibration module calculated for the accelerometer-registered responses,

$t$ - time vector,

$f-$ frequency vector,

$g(t, f)$ - dependency presenting the change of vibration modules in the time and frequency domain.

By the application of Cartesian product formula for vectors $t(i)$ and $f(i)$, which represent discrete time and frequency variable of an analyzed response, matrixes (4) were calculated. They form a point net on the TF plane ( $T$ - time, $F-$ frequency).

$$
(T(i, j), F(i, j))=(t(i), f(j))
$$

where:

$i, j-$ number of points of time and frequency vector,

$T, F$ - matrixes of time $\mathrm{T}(\mathrm{i}, \mathrm{j})$ and frequency $\mathrm{F}(\mathrm{i}, \mathrm{j})$ calculated with the use of Cartesian product formula for vectors $t(i)$ i $f(j)$.

Next, the points of matrix FFT (i,j), which represent the spectra of amplitude vibrations registered by accelerometer, have been marked on this defined net. Therefore, timefrequency dynamic characteristics $2 \mathrm{D}$ are the planes drawn in space defined by the set of points (5).

$$
\{T(i, j), F(i, j), F F T(i, j)\}
$$

The pcolor function (2D) or surf function (3D) has been used to draw the abovementioned planes by the use of Matlab program. In so doing, on the Figures 12-14 the change of response amplitude in the time and frequency domain was introduced, where the change of response amplitude in the time domain has been marked with a color on an assumptive color scale. 

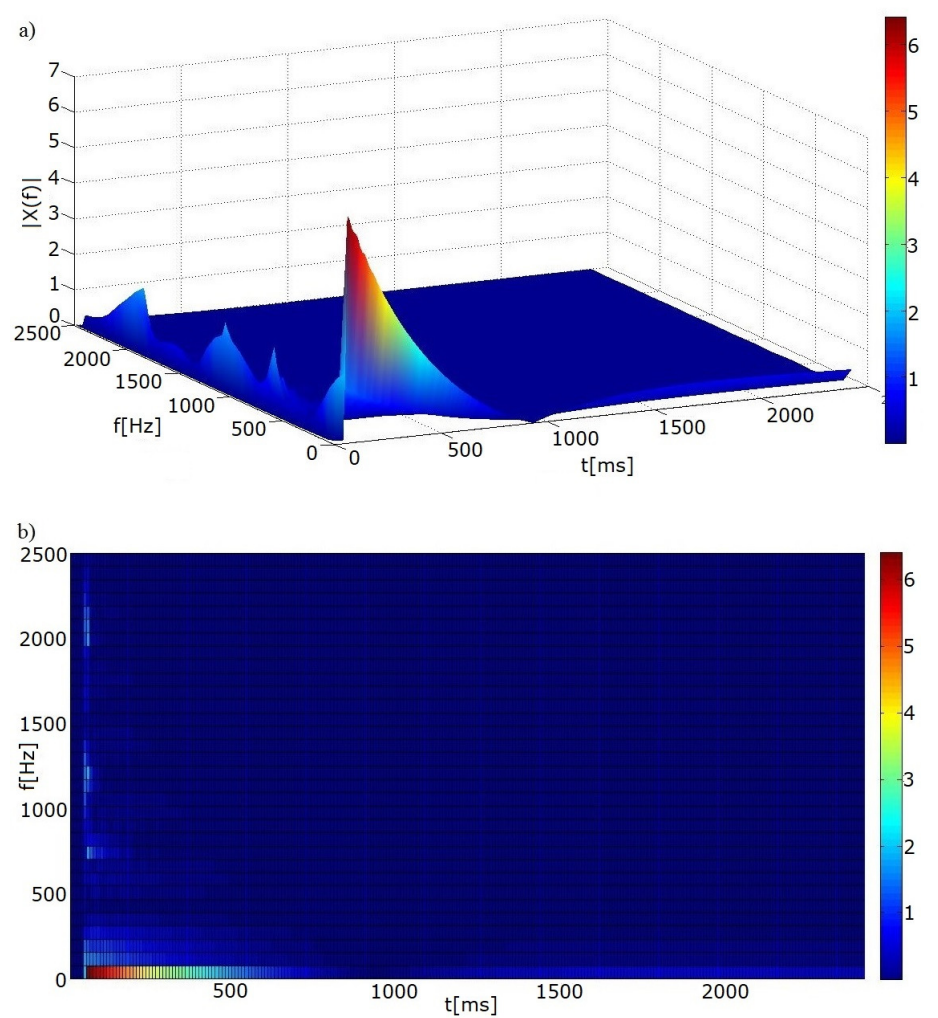

Fig. 12 Time-frequency dynamic characteristics 3D (a) and 2D (b) for a plate with no welded joints $(0)$ for the impact made by an modal hammer with a metal head
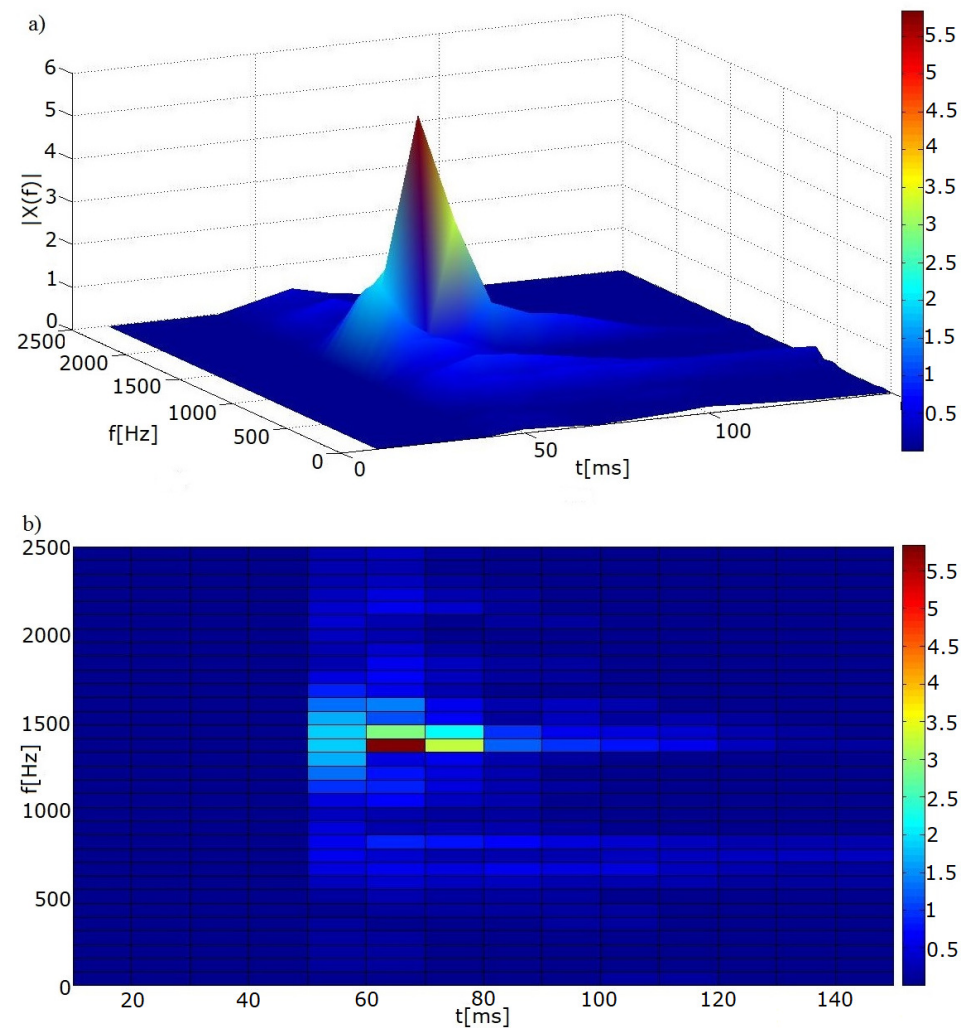

Fig. 13 Time-frequency dynamic characteristics 3D (a) and 2D (b) for a welded plate with non-defected welded joint (2202) for the impact made by an impact modal with a metal head 

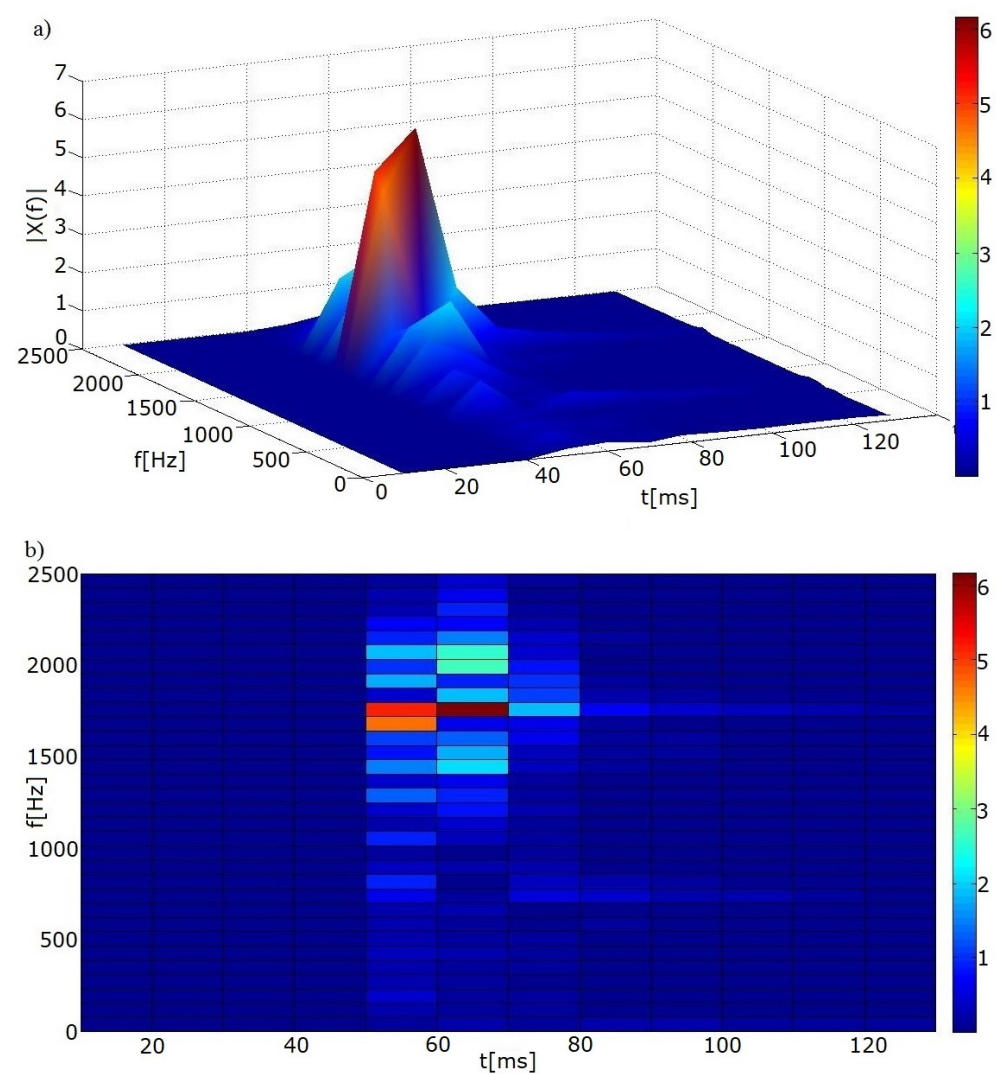

Fig. 14 Time-frequency dynamic characteristics 3D (a) and 2D (b) for a welded plate with a fault (lack of side fusion [2127]) for the impact made by impact hammer with a metal tip

The characteristics presented in Figures 12-14 clearly show that presentation of response on both the time and frequency domains allows assessing whether the tested configuration remains linear and, at the same time, indirectly enabling to estimate the quality of a welded joint. In this instance, the measure of the quality of a welded joint may be the dispersion of colors on the characteristics presented above. By comparing the characteristics from Figure 12 (a plate without a welded joint) to the characteristics from Figures 13 and 14 (plates with welded joints) there can be noticed that the plate without a welded joint practically does not contain dispersion, and all the other plates do. Even the plate with the correct weld (Figure 13) has dispersion, so each weld, even a high quality one, changes the mechanical properties of the material. However if the quality of the weld is worse, the dispersion is larger, which represents a greater non-linearity compared to a plate with edge bonding.

\section{TIME WINDOW METHOD IN TIME-FREQUENCY ANALYSIS}

The time window method enables simulation of concurrent signal analysis in the field of frequency and time, similarly to the method presented in chapter 4 . This opportunity is often used in the analysis of technical systems dynamics, especially electrical or electronic ones. It is used in mechanical systems testing very rarely (Muc, et. al., 2018). In case of time window method, the key element which influences the quality of results is a proper selection of the type and width of the time window. Then the FFT analysis (Fast Fourier Transform) is carried out for each of windows. 
The time windows method was used in conducted tests in order to analyze the responses which were recorded by piezoelectric accelerometers. The tests were done for rectangular window in terms of which various time intervals were checked. The decision to choose the rectangular window comes from the willingness to reduce the distortions of recorded course of response to the least extent. This kind of situation often takes place in case of more complex windows. Then at the cost of accepted changes introduced from the signal, it is possible to eliminate the part of distortions through the window. Conducted tests aimed at the optimal coverage of spectrums characteristic changes which determine the quality of welds and possible defects. The optimal width of the window was chosen as $10 \mathrm{~ms}$ for analyzed plates (Muc, et. al., 2018). The preliminary analysis of spectrums shows that most diagnostic information comes from first ten time windows which mean about $100 \mathrm{~ms}$ of analyzed response registered by accelerometer.

The amplitude spectrums are characterized by similar character of distribution, especially in terms of harmonics, however, important diagnostic information is given by the spectrum of higher harmonics, which analysis requires additional mathematical operations. Proposed parameter to compare the amplitude spectrums calculated for each window of the same sample and to evaluate the nonlinearity resulting from welded joint in a plate was the mean distributions of amplitude spectrums. The mean value is a measure of descriptive statistics which was calculated from the equation (4).

$$
\overline{|v|}=\frac{\sum_{i=0}^{N}\left|v\left(f_{i}\right)\right|}{N}
$$

where:

$|v|$ - the mean of amplitude spectrum velocity calculated for the time window,

$f_{i}$ - the frequency of ith harmonic,

$N$ - the number of harmonics.

The preliminary tests have shown that the analysis of only mean values is not sufficient. However, the relevant diagnostic information about the quality of welded joint is given by mean value distribution for the spectrums obtained from the whole response of time windows. Fig. 15 shows some examples of amplitude spectrums taken from chosen time windows and the values calculated for them. The distribution shown in Fig. 16, presents the alteration of the mean value calculated for the amplitude spectrum in given window in function of window number for a chosen welded plate. Presented characteristics show that the mean distribution for windows calculated for the sample without the weld (marked as number 0 ) differs significantly in comparison to mean distribution of other samples with weld or samples with a damage in welded joint (marked as: 2202, 2132, 2127). This difference is especially applicable in terms of the level of mean values and cyclic value change between local maximums and minimums. It is also possible to notice that the mean value distributions for the samples with the weld and a damage are characterized by lower value than for the plates with weld and without damages.

The comparison analysis of amplitude spectrums, calculated for each window, shows nonlinearities of structures of damaged welded plates. If the quality of welded joint is worse, the nonlinearities are stronger, that is when the defect is shown in the welded joint. 

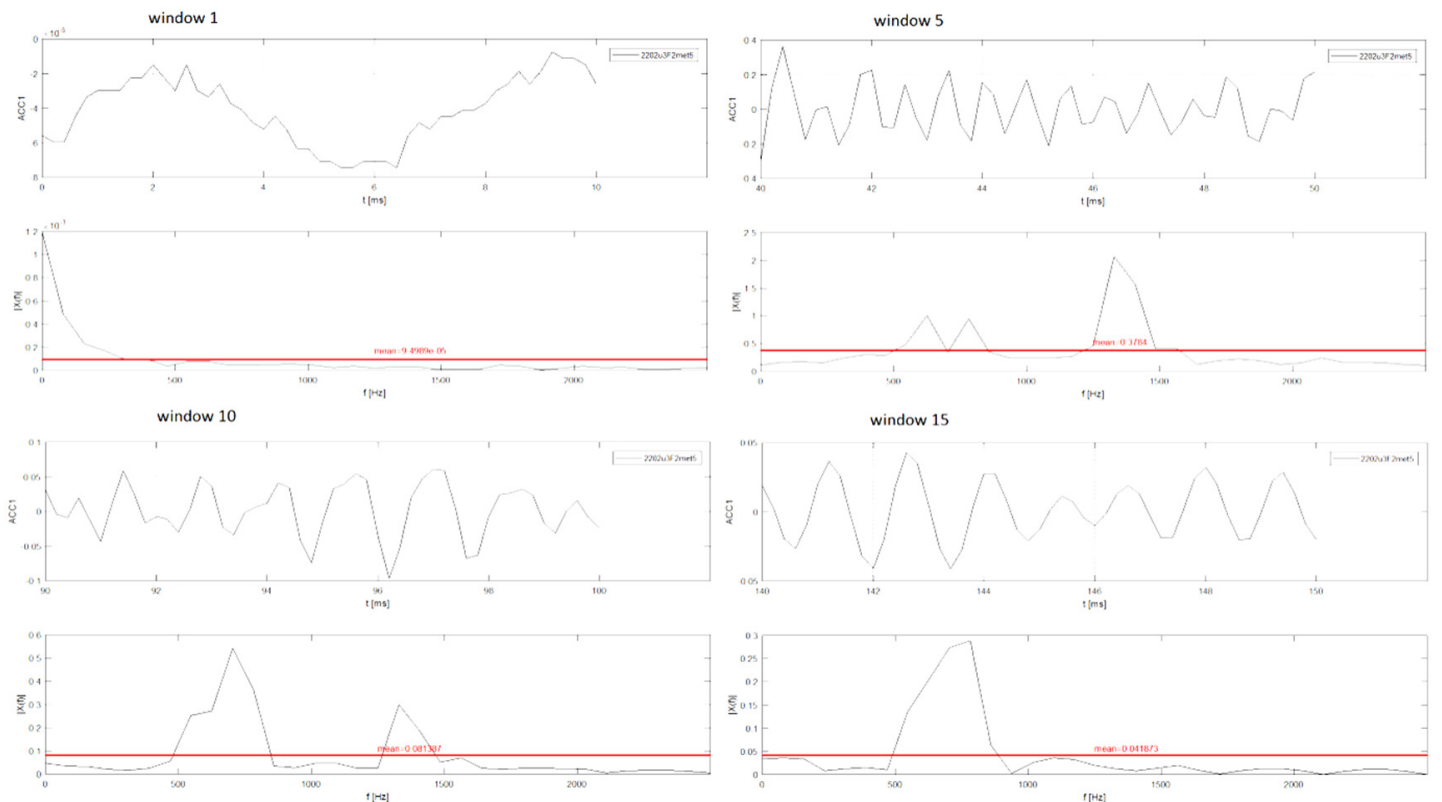

Fig. 15 Amplitude spectrums in chosen windows with the designated mean value

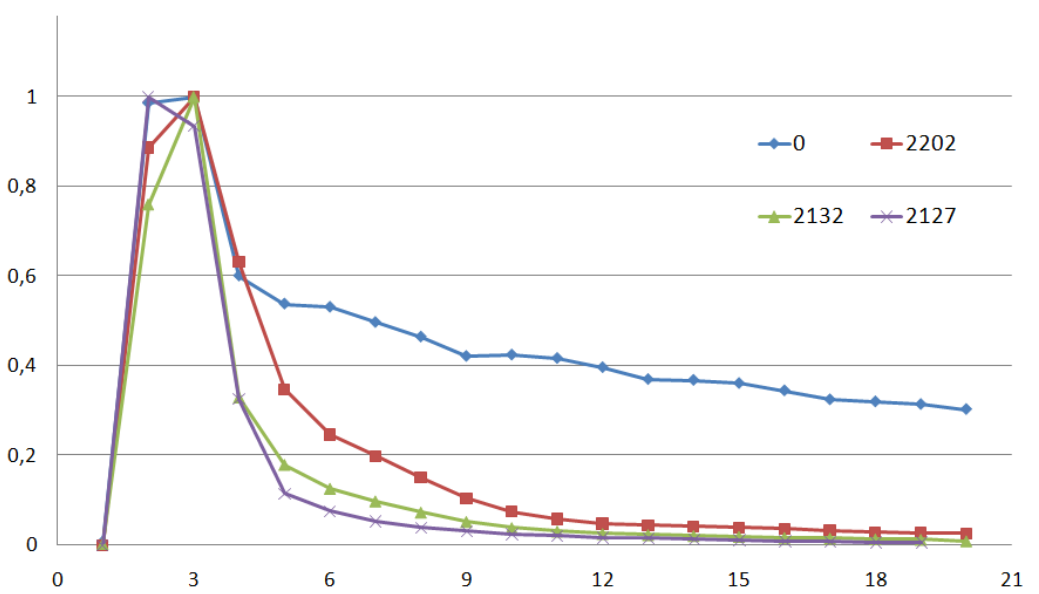

Fig. 16 Standardized distribution of mean value of the spectrums for windows in function of window number

\section{CONCLUSIONS}

The presented analysis methods - benchmarks of the dynamic characteristics of marine thin-walled structures allows concluding that it will be possible to detect the damages of the welded joint in autonomic maritime structural health monitoring. As expected, for a plate made of one material and without weld, the damping decrement distribution obtained for different approximating functions is practically linear and parallel to the characteristic of the reference value. In the other cases, i.e. for plates with welded joints, the characteristics of damping decrement clearly curve and tend to intersect with the characteristic of reference decrement of the reference. The results show that the characteristics of damping decrement curve faster for the plates which weld has a fault.

Diagnostic data acquired as a result of the analysis of time-frequency dynamic characteristics also allows to assess the condition and quality of joints. Due to the availability of image processing functions in numerous computational programs, it is possible to apply the proposed time-frequency dynamic characteristics 2D or 3D in 
order to detect any joint decrements. This can be performed by the use of automatic construction monitoring systems like SHM. Similarly to the use of the mean value distribution from time windows for the evaluation of welded joints, the use of timefrequency characteristics allowed us to analyze responses from accelerometers in both time and frequency domain.

The proposed quality assessment method requires further tests as well as the introduction of a third dimension such as a module variable of calculated spectral amplitude. Then, apart from applying the data referring to time-frequency dynamic characteristic, there would be a possibility to use also the information about the intensity of changes in amplitude spectra.

\section{REFEENCES}

Abrantes, R. F. D. (2014). Electronic System for Non-Destructive Testing using Eddy Currents Array Probes, Tecnico Lisboa.

Dae-Seung, C., Tae-Muk C., Jin-Hyeong, K. and Nikola, V. (2016). Structural intensity analysis of stepped thickness rectangular plates utilizing the finite element method, Thin-Walled Structures, 109, pp. 1-12.

Jiawei, Y., Songlin Z., Hoang P. and Tie C. (2018). Reliability modeling of multi-state degraded repairable systems and its applications to automotive systems. Quality and Reliability Engineering International, 34 (3), pp. 459-474.

Krause, M., Dackermann, U., Jianchun, L. (2015). Elastic wave modes for the assessment of structural timber: Ultrasonic echo for building elements and guided waves for pole and pile structures. Journal of Civil Structural Health Monitoring, 5(2), pp. 221-249.

Masayasu, O. (2016). Innovative AE and NDT Techniques for On-Site Measurement of Concrete and Masonry Structures. Springer.

Montewka, J., Ehlers, S., Goerlandt, F., Hinz, T., Tabri, K. and Kujala, P. A. (2014). framework for risk assessment for maritime transportation systems-A case study for open sea collisions involving RoPax vessels. Reliability Engineering and System Safety,124, pp. 142-157.

Muc, A., Murawski, L. and Szeleziński, A. (2018). Methods of cracks detection in marine structures' welded joints based on signals' time waveform analysis. Brodogradnja/Shipbilding, 69 (3), pp. 43-59.

Murawski, L., Ostachowicz, W., Opoka, S., Mieloszyk, M. and Majewska, K. (2012). Practical application of monitoring system based on optical sensors for marine constructions. Key Engineering Materials, 518, pp. 261-270.

Mvola, B., Suoranta, R. (2014). Real Time Non-Destructive Testing Methods of Welding, ReaserchGate.

Onqpeng, J. M. C., Oreta, A. and Hirose S. (2018). Monitoring Damage Using Acoustic Emission Source Location and Computational Geometry in Reinforced Concrete Beams, MDPI.

Opoka, S., Murawski, L., Wandowski, T., Malinowski, P. and Ostachowicz, W. (2013). Static - Strain Level Change Together with Detection of Transient Signal as Damage Indicator for Truss and Fame Structures. Strain, 49(4), pp. 287-298.

Recho N. (2012). Fracture Mechanics and Crack growth, John WILEY.

Runnemalm, A. (2012). Vibration Induced Disturbances in Automatic Non-destructive Testing, 18th World Conference on Nondestructive Testing.

Senjanović, I., Vladimir, N., Tomić, M., Hadžić, N. and Malenica, Š. (2014). Some aspects of structural modelling and restoring stiffness in hydroelastic analysis of large container ships. Ships and Offshore Structures, 9(2), pp. 199-217.

Szeleziński, A., Muc, A. and Murawski, L. (2017). Analysis concerning changes of structure damping in welded joints diagnostics. Journal of KONES Powertrain and Transport, 24(4), pp. 314-320.

Szeleziński, A., Murawski, L. and Muc, A. (2016). Analysis of ability to detect defects in welding structures with usage of dynamic characteristics spectrums. Journal of KONES Powertrain and Transport, 23(2), pp. 365-372. 
Vizentin, G., Vukelić, G. (2017). Common failures of ship propulsion shafts. Pomorstvo, 31(2), pp. 85-90.

Vukelić, G., Brnić, J. (2016). Predicted Fracture Behaviour of Shaft Steels with Improved Corrosion Resistance, Metals, 6(2), pp. 1-9.

Abstract: Among structural health monitoring (SHM) methods of thin-walled structures, a vibrodiagnostic method is one of the most promising. The accelerometer recorded responses provide diagnostic information that requires mathematical processing to extract the essential dynamic characteristics. The authors have been looking for new parameters - diagnostic benchmarks which can be applied to nondestructive, automatic testing of thin-walled marine structures (especially their welded joints) like ship hulls. All characteristics have been based on recorded data generated during the vibration tests of welded joints with and without failures. For this purpose, the authors proposed method based on: FFT windowing analysis, benchmark with using $2 \mathrm{D}$ or $3 \mathrm{D}$ time - frequency dynamic characteristics and the determination of damping decrement in function of time. The work presents the algorithm and exemplary results obtained from the application of proposed method to several selected sample plates with different type of welds.

Keywords: thin-walled structures, welded joints, SHM, spectrum analysis, damping decrement 